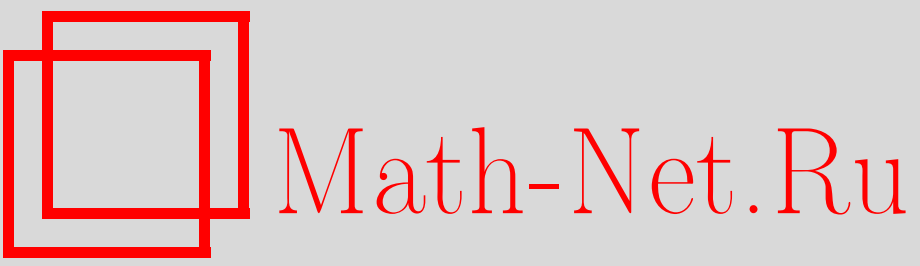

Ж. А. Балкизов, Об априорной оценке решения задачи Трикоми для одного уравнения смешанного типа второго порядка, Итоги науки и техн. Сер. Соврем. мат. и ее прил. Темат. обз., 2019, том 167, 14-20

DOI: https://doi.org/10.36535/0233-6723-2019-167-14-20

Использование Общероссийского математического портала Math-Net.Ru подразумевает, что вы прочитали и согласны с пользовательским соглашением

http://www.mathnet.ru/rus/agreement

Параметры загрузки:

IP : 54.237 .59 .107

26 апреля 2023 г., 12:23:54 


\title{
ОБ АПРИОРНОЙ ОЦЕНКЕ РЕШЕНИЯ ЗАДАЧИ ТРИКОМИ ДЛЯ ОДНОГО УРАВНЕНИЯ СМЕШАННОГО ТИПА ВТОРОГО ПОРЯДКА
}

\author{
(c) 2019 г. Ж. А. БАЛКИЗОВ
}

\begin{abstract}
АннотАция. В работе доказана теорема об априорной оценке решения задачи Трикоми для уравнения смешанного типа второго порядка с оператором Геллерстедта в области гиперболичности. Из полученной априорной оценки вытекает единственность регулярного решения исследуемой задачи.
\end{abstract}

Ключевъе слова: уравнение Пуассона, уравнение Геллерстедта, уравнение смешанного типа, задача Трикоми, задача Коши, априорная оценка.

\section{ON A PRIORI ESTIMATES OF SOLUTIONS OF THE TRICOMI PROBLEM FOR A CERTAIN MIXED-TYPE SECOND-ORDER EQUATION}

\author{
(c) 2019 G. A. BALKIZOV
}

\begin{abstract}
In this paper, a theorem on a priori estimates of solutions of the Tricomi problem for a second-order, mixed-type equation with the Gellerstedt operator in the hyperbolicity domain is proved. The a priori estimate obtained implies the uniqueness of the regular solution of the problem considered.

Keywords and phrases: Poisson equation, Gellerstedt equation, mixed-type equation, Tricomi problem, Cauchy problem, a priori estimate.
\end{abstract}

AMS Subject Classification: 35M10, 35M13

1. Введение. Одним из первых работ, посвященных фундаментальным исследованиям по теории уравнений смешанного эллиптико-гиперболического типа второго порядка были работы Ф. Трикоми. Так, в [29] была поставлена и исследована краевая задача для уравнения (1) в стандартной смешанной области $D$, ограниченной при $y \geqslant 0$ гладкой кривой $\Gamma$ с концами в точках $A$ и $B$ (точки $A$ и $B$ не совпадают и лежат на оси $O x$ ), а при $y \leqslant 0$-характеристиками $A C$ и $B C$ уравнения

$$
y u_{x x}+u_{y y}=0 .
$$

В $[32,33]$ результаты $\Phi$. Трикоми были обобщены на случай уравнения

$$
y^{m} u_{x x}+u_{y y}+c(x, y) u=F(x, y),
$$

где $m$ - нечетное натуральное число.

С целью упрощения исследований по теории краевых задач для уравнений смешанного типа, в $[7,9,17]$ вместо уравнений (1) и (2) была предложена более простая модель смешанного 
уравнения - уравнение Лаврентьева-Бицадзе

$$
\operatorname{sgn} y u_{x x}+u_{y y}=F(x, y) .
$$

Различные краевые задачи для модельных и общих уравнений смешанного гиперболо-эллиптического типа второго порядка были исследованы в $[2,10,12-15,19,20,22,26,31]$. Подробный обзор литературы по проблеме краевых задач для уравнений смешанного типа содержатся в монографиях $[7-9,11,16,18,23-25,27-29]$. Вопросам применения теории краевых задач для уравнений смешанного типа к проблемам околозвуковой и сверхзвуковой газовой динамики посвящены монографии $[5,6,30]$.

В большинстве перечисленных работ исследования проводились по краевым задачам для уравнений смешанного типа, порядок вырождения которых из гиперболической и эллиптической частей области совпадали.

В данной работе исследована краевая задача Трикоми для уравнения смешанного типа, которое совпадает с уравнением Пуассона в области эллиптичности и с уравнением Геллерстедта в области гиперболичности. Получена априорная оценка решения задачи, из которой следует единственность регулярного решения исследуемой задачи, а также существование слабого решения задачи, сопряженной к исследуемой.

2. Постановка задачи. На евклидовой плоскости независимых переменных $x$ и $y$ рассмотрим уравнение

$$
0= \begin{cases}u_{x x}+u_{y y}+f, & y>0 \\ (-y)^{m} u_{x x}-u_{y y}, & y<0\end{cases}
$$

где $u=u(x, y)$ - искомая функция, $f=f(x, y)$ - заданная функция, $m$ - заданное положительное число.

Уравнение (3) рассматривается в области $\Omega$, ограниченной прямоугольником $\Omega_{2}$ с вершинами в точках $A=(0,0), A_{0}=(0, h), B_{0}=(r, h)$ и $B=(r, 0)$ при $y>0$, а также характеристиками

$$
A C: x-\frac{2}{m+2}(-y)^{(m+2) / 2}=0, \quad C B: x+\frac{2}{m+2}(-y)^{(m+2) / 2}=r
$$

уравнения (3), выходящими из общей точки $C=\left(\frac{r}{2},-\left[\frac{(m+2) r}{4}\right]^{2 /(m+2)}\right)$ и проходящими через точки $A$ и $B$ соответственно. Пусть $\Omega_{1}=\Omega \cap\{y<0\}, \Omega_{2}=\Omega \cap\{y>0\}, J=\{(x, y)$ : $0<x<r, y=0\}$ - интервал $A B$ прямой $y=0 ; \Omega=\Omega_{1} \cup \Omega_{2} \cup J$.

В области $\Omega$ уравнение (3) является уравнением смешанного типа: оно гиперболично в области $\Omega_{1}$ и эллиптично в области $\Omega_{2}$.

Определение 1. Регулярным в области $\Omega$ решением уравнения (3) назовем функцию $u=$ $u(x, y)$ из класса $C(\bar{\Omega}) \cap C^{1}(\Omega) \cap C^{2}\left(\Omega_{1} \cup \Omega_{2}\right)$, при подстановке которой уравнение (3) обращается в тождество.

В работе исследуется задача Трикоми для уравнения (3) в следующей постановке.

Задача 1. Найти регулярное в области $\Omega$ решение $u=u(x, y)$ уравнения $(3)$, удовлетворяющее краевым условиям

$$
\begin{gathered}
u(0, y)=\varphi_{1}(y), \quad u(r, y)=\varphi_{2}(y), \quad 0 \leqslant y \leqslant h, \\
u(x, h)=\varphi_{3}(x), \quad 0 \leqslant x \leqslant r, \\
\left.u(x, y)\right|_{A C}=\psi(x), \quad 0 \leqslant x \leqslant r / 2,
\end{gathered}
$$

где $\varphi_{1}(y), \varphi_{2}(y), \varphi_{3}(x), \psi(x)$ - заданные функции из соответствующих классов: $\varphi_{1}(y), \varphi_{2}(y) \in$ $C[0, h], \varphi_{3}(x) \in C[0, r], \psi(x) \in C^{3}[0, r / 2]$ и выполнены условия согласования:

$$
\varphi_{1}(0)=\psi(0), \quad \varphi_{1}(h)=\varphi_{3}(0), \quad \varphi_{2}(h)=\varphi_{3}(r) .
$$


3. Фундаментальное соотношение. В дальнейшем будем предполагать, что решение задачи (3)-(6) существует. Введем обозначения

$$
\begin{gathered}
u(x, 0)=\tau(x), \quad 0 \leqslant x \leqslant r \\
u_{y}(x, 0)=\nu(x), \quad 0<x<r .
\end{gathered}
$$

Решение задачи Коши (7)-(8) для уравнения (3) в области $\Omega_{1}$ выписывается по формуле [9, с. 45]:

$$
\begin{array}{r}
u(x, y)=\gamma_{1} \int_{0}^{1} \tau\left[x+\frac{2}{m+2}(-y)^{(m+2) / 2}(2 t-1)\right] t^{\beta-1}(1-t)^{\beta-1} d t+ \\
\quad+\gamma_{2} y \int_{0}^{1} \nu\left[x+\frac{2}{m+2}(-y)^{(m+2) / 2}(2 t-1)\right] t^{-\beta}(1-t)^{-\beta} d t,
\end{array}
$$

где

$$
\beta=\frac{m}{2(m+2)}, \quad \gamma_{1}=\frac{\Gamma(2 \beta)}{\Gamma^{2}(\beta)}, \quad \gamma_{2}=\frac{\Gamma(2-2 \beta)}{\Gamma^{2}(1-\beta)} .
$$

Потребовав, чтобы функция (9) удовлетворяла условию (6), найдем

$$
\gamma_{1} \int_{0}^{1} \tau[2 t x] t^{\beta-1}(1-t)^{\beta-1} d t-\gamma_{2}\left(\frac{x}{1-2 \beta}\right)^{1-2 \beta} \int_{0}^{1} \nu[2 t x] t^{-\beta}(1-t)^{-\beta} d t=\psi(x) .
$$

Введя в подынтегральных выражениях последнего равенства новую переменную интегрирования $z=2 t x$ и поменяв $2 x$ на $x$ в полученном равенстве, придем к равенству

$$
\gamma_{1} x^{1-2 \beta} \int_{0}^{x} \frac{\tau(t) t^{\beta-1}}{(x-t)^{1-\beta}} d t-\frac{\gamma_{2}}{(2-4 \beta)^{1-2 \beta}} \int_{0}^{x} \frac{\nu(t) t^{-\beta}}{(x-t)^{\beta}} d t=\psi\left(\frac{x}{2}\right) .
$$

Пользуясь определением оператора $D_{a x}^{\alpha}$ дробного (в смысле Римана-Лиувилля) интегродифференцирования порядка $|\alpha|$ с началом в точке $a$ (см. $[21$, с. 28], перепишем равенство (10) в виде

$$
\Gamma(\beta) \gamma_{1} x^{1-2 \beta} D_{0 x}^{-\beta} t^{\beta-1} \tau(t)-\frac{\Gamma(1-\beta) \gamma_{2}}{(2-4 \beta)^{1-2 \beta}} D_{0 x}^{\beta-1} t^{-\beta} \nu(t)=\psi\left(\frac{x}{2}\right) .
$$

Применим к обеим частям (11) оператор $D_{0 x}^{1-\beta}$ :

$$
x^{-\beta} \nu(x)=\gamma_{3} D_{0 x}^{1-\beta} t^{1-2 \beta} D_{0 t}^{-\beta} s^{\beta-1} \tau(s)-\gamma_{4} D_{0 x}^{1-\beta} \psi\left(\frac{t}{2}\right),
$$

где

$$
\gamma_{3}=\frac{\Gamma(2 \beta) \Gamma(1-\beta)}{\Gamma(\beta) \Gamma(2-2 \beta)}(2-4 \beta)^{1-2 \beta}, \quad \gamma_{4}=\frac{\Gamma(\beta) \gamma_{3}}{\Gamma(2 \beta)} .
$$

Воспользуемся далее свойством композиции операторов дробного интегродифференцирования с одинаковыми началами (см. [21, с. 44]):

$$
D_{0 x}^{1-\beta} t^{1-2 \beta} D_{0 t}^{-\beta} s^{\beta-1} \tau(s)=x^{-\beta} D_{0 x}^{1-2 \beta} \tau(t) .
$$

С учетом (13) из (12) находим

$$
\nu(x)=\gamma_{3} D_{0 x}^{1-2 \beta} \tau(t)-\gamma_{4} x^{\beta} D_{0 x}^{1-\beta} \psi\left(\frac{t}{2}\right) .
$$

Соотношение (14) есть фундаментальное соотношение между функциями $\tau(x)$ и $\nu(x)$, принесенное из области $\Omega_{1}$ на линию $y=0$. 
4. Априорная оценка решения. В дальнейших рассуждениях используем нормы

$$
\|v(x, y)\|_{0}^{2}=\int_{\Omega_{2}} v^{2}(x, y) d x d y, \quad\|g(x)\|_{0}^{2}=\int_{0}^{r} g^{2}(x) d x .
$$

С учетом полученного выше фундаментального соотношения (14) между функциями $\tau(x)$ и $\nu(x)$, в области $\Omega_{2}$ приходим к задаче нахождения решения уравнения Пуассона, удовлетворяющего условиям (4), (5) и условию

$$
u_{y}(x, 0)=\gamma_{3} D_{0 x}^{1-2 \beta} u(t, 0)-\gamma_{4} x^{\beta} D_{0 x}^{1-\beta} \psi\left(\frac{t}{2}\right) .
$$

Введем вспомогательную область $\Omega_{2 \varepsilon}=\{(x, y): \varepsilon<x<r-\varepsilon, \varepsilon<y<h-\varepsilon, \varepsilon>0\}$. Умножим уравнение (3) при $y>0$ на функцию $u(x, y)$ и проинтегрируем полученное равенство по вспомогательной области $\Omega_{2 \varepsilon}$. Тогда для любой функции $f(x, y) \in L_{2}(\Omega)$ имеем:

$$
\begin{aligned}
\int_{\Omega_{2 \varepsilon}} u(x, y)\left[u_{x x}(x, y)+u_{y y}(x, y)\right] d x d y & =\int_{\Omega_{2 \varepsilon}}\left[\frac{\partial}{\partial x}\left(u u_{x}\right)+\frac{\partial}{\partial y}\left(u u_{y}\right)\right] d x d y- \\
& -\int_{\Omega_{2 \varepsilon}}\left[u_{x}^{2}(x, y)+u_{y}^{2}(x, y)\right] d x d y=-\int_{\Omega_{2 \varepsilon}} u(x, y) f(x, y) d x d y .
\end{aligned}
$$

Применим к последнему равенству формулу Грина:

$$
\int_{\Gamma_{2 \varepsilon}}\left(u u_{x}\right) d y-\left(u u_{y}\right) d x-\int_{\Omega_{2 \varepsilon}}\left[u_{x}^{2}(x, y)+u_{y}^{2}(x, y)\right] d x d y=-\int_{\Omega_{2 \varepsilon}} u(x, y) f(x, y) d x d y,
$$

где $\Gamma_{2 \varepsilon}-$ граница вспомогательной области $\Omega_{2 \varepsilon}$.

Перейдем в равенстве $(16)$ к пределу при $\varepsilon \rightarrow 0$. Легко заметить, что при этом область $\Omega_{2 \varepsilon}$ переходит в область $\Omega_{2}$, а граница $\Gamma_{2 \varepsilon}$ переходит в границу $\Gamma_{2}$ области $\Omega_{2}$. Тогда из (16) получим

$$
\int_{\Gamma_{2}}\left(u u_{x}\right) d y-\left(u u_{y}\right) d x-\int_{\Omega_{2}}\left[u_{x}^{2}(x, y)+u_{y}^{2}(x, y)\right] d x d y=-\int_{\Omega_{2}} u(x, y) f(x, y) d x d y .
$$

Вычислим интеграл по границе $\Gamma_{2}$. В случае однородных условий (4)-(5) находим

$$
\begin{aligned}
\int_{\Gamma_{2}}\left(u u_{x}\right) d y-\left(u u_{y}\right) d x= & \int_{0}^{r}\left[u(x, h) u_{y}(x, h)-u(x, 0) u_{y}(x, 0)\right] d x+ \\
& +\int_{0}^{h}\left[u(r, y) u_{x}(r, y)-u(0, y) u_{x}(0, y)\right] d y=-\int_{0}^{r} u(x, 0) u_{y}(x, 0) d x .
\end{aligned}
$$

С учетом (18) равенство (17) перепишется в следующем виде:

$$
\int_{0}^{r} u(x, 0) u_{y}(x, 0) d x+\left\|u_{x}\right\|_{0}^{2}+\left\|u_{y}\right\|_{0}^{2}=\int_{\Omega_{2}} u(x, y) f(x, y) d x d y .
$$

Подставляя значение $u_{y}(x, 0)$ из $(15)$ в $(19)$, приходим к равенству

$$
\begin{aligned}
\left\|u_{x}\right\|_{0}^{2}+\left\|u_{y}\right\|_{0}^{2}+\gamma_{3} \int_{0}^{r} u(x, 0) D_{0 x}^{1-2 \beta} & u(t, 0) d x- \\
& \quad-\gamma_{4} \int_{0}^{r} u(x, 0) x^{\beta} D_{0 x}^{1-\beta} \psi\left(\frac{t}{2}\right) d x=\int_{\Omega_{2}} u(x, y) f(x, y) d x d y .
\end{aligned}
$$


Оценим слагаемые, входящие в равенство (20). Применяя [1, лемма 1.1], убеждаемся в справедливости неравенства

$$
\begin{gathered}
\gamma_{3} \int_{0}^{r} \tau(x) D_{0 x}^{1-2 \beta} \tau(t) d x \geqslant \frac{\gamma_{3}}{2} \int_{0}^{r} \partial_{0 x}^{1-2 \beta} \tau^{2}(t) d x=\frac{\gamma_{3}}{2} \int_{0}^{r} D_{0 x}^{1-2 \beta} \tau^{2}(t) d x= \\
=\frac{\gamma_{3}}{2 \Gamma(2 \beta)} \int_{0}^{r} \frac{d}{d x} \int_{0}^{x} \frac{\tau^{2}(t) d t}{(x-t)^{1-2 \beta}} d x=\frac{\gamma_{3}}{2 \Gamma(2 \beta)} \int_{0}^{r} \frac{\tau^{2}(t)}{(r-t)^{1-2 \beta}} d t \geqslant \frac{\gamma_{3} r^{2 \beta-1}}{2 \Gamma(2 \beta)} \int_{0}^{r} \tau^{2}(x) d x,
\end{gathered}
$$

а использование $\varepsilon$-неравенства дает

$$
-\gamma_{4} \int_{0}^{r} \tau(x) x^{\beta} D_{0 x}^{1-\beta} \psi\left(\frac{t}{2}\right) d x \geqslant \int_{0}^{r}\left[-\gamma_{4} \varepsilon_{1} \tau^{2}(x)-\frac{\gamma_{4}}{4 \varepsilon_{1}} \chi^{2}(x)\right] d x=-\gamma_{4} \varepsilon_{1}\|\tau(x)\|_{0}^{2}-\frac{\gamma_{4}}{\varepsilon_{1}}\|\chi(x)\|_{0}^{2},
$$

где $\chi(x)=x^{\beta} D_{0 x}^{1-\beta} \psi(t / 2)$, а $\varepsilon_{1}$ - достаточно малое положительное число. Аналогично,

$$
\int_{\Omega_{2}} u(x, y) f(x, y) d x d y \leqslant \int_{\Omega_{2}}\left[\varepsilon_{2} u^{2}(x, y)+\frac{1}{4 \varepsilon_{2}} f^{2}(x, y)\right] d x d y=\varepsilon_{2}\|u(x, y)\|_{0}^{2}+\frac{1}{4 \varepsilon_{2}}\|f(x, y)\|_{0}^{2},
$$

где $\varepsilon_{2}$ - достаточно малое положительное число. С учетом полученных выше неравенств из (20) имеем

$$
\left(\frac{\gamma_{3} r^{2 \beta-1}}{2 \Gamma(2 \beta)}-\gamma_{4} \varepsilon_{1}\right) \int_{0}^{r} \tau^{2}(x) d x-\varepsilon_{2}\|u\|_{0}^{2}+\left\|u_{x}\right\|_{0}^{2}+\left\|u_{y}\right\|_{0}^{2} \leqslant \frac{\gamma_{4}}{4 \varepsilon_{1}}\|\chi(x)\|_{0}^{2}+\frac{1}{4 \varepsilon_{2}}\|f(x, y)\|_{0}^{2} .
$$

Выберем значение постоянной $\varepsilon_{1}$ так, чтобы

$$
\varepsilon_{1}<\frac{\gamma_{3} r^{2 \beta-1}}{2 \gamma_{4} \Gamma(2 \beta)}
$$

Тогда из последнего неравенства получим

$$
-\varepsilon_{2}\|u\|_{0}^{2}+\left\|u_{x}\right\|_{0}^{2}+\left\|u_{y}\right\|_{0}^{2} \leqslant \frac{\gamma_{4}}{4 \varepsilon_{1}}\|\chi(x)\|_{0}^{2}+\frac{1}{4 \varepsilon_{2}}\|f(x, y)\|_{0}^{2} .
$$

Оценим далее $\left\|u_{x}\right\|_{0}^{2}$. Для этого заметим, что

$$
u^{2}(x, y)=\left(\int_{0}^{x} u_{s}(s, y) d s\right)^{2} \leqslant x \int_{0}^{x} u_{s}^{2}(s, y) d s \leqslant x \int_{0}^{r} u_{x}^{2}(x, y) d x .
$$

Проинтегрируем обе части (22) сначала по $x$ от 0 до $r$, а затем по $y$ от 0 до $h$. Будем иметь

$$
\begin{gathered}
\int_{0}^{r} u^{2}(x, y) d x \leqslant \frac{r^{2}}{2} \int_{0}^{r} u_{x}^{2}(x, y) d x \\
\int_{0}^{h}\left(\int_{0}^{r} u^{2}(x, y) d x\right) d y=\int_{\Omega_{2}} u^{2}(x, y) d x d y \leqslant \frac{r^{2}}{2} \int_{0}^{h}\left(\int_{0}^{r} u_{x}^{2}(x, y) d x\right) d y=\frac{r^{2}}{2} \int_{\Omega_{2}} u_{x}^{2}(x, y) d x d y,
\end{gathered}
$$

откуда

$$
\frac{2}{r^{2}}\|u\|_{0}^{2} \leqslant\left\|u_{x}\right\|_{0}^{2}
$$

Аналогично, интегрируя неравенство

$$
u^{2}(x, y)=\left(-\int_{y}^{h} u_{s}(x, s) d s\right)^{2} \leqslant y \int_{0}^{h} u_{y}^{2}(x, y) d y
$$


сначала по $y$ от 0 до $h$, а затем по $x$ от 0 до $r$, приходим к оценке

$$
\frac{2}{h^{2}}\|u\|_{0}^{2} \leqslant\left\|u_{y}\right\|_{0}^{2}
$$

С учетом оценок (23) и (24) из (21) получаем

$$
\left(\frac{2}{r^{2}}+\frac{2}{h^{2}}-\varepsilon_{2}\right)\|u\|_{0}^{2} \leqslant \frac{\gamma_{4}}{4 \varepsilon_{1}}\|\chi(x)\|_{0}^{2}+\frac{1}{4 \varepsilon_{2}}\|f(x, y)\|_{0}^{2} .
$$

Выбирая произвольную постоянную $0<\varepsilon_{2}<2 / r^{2}+2 / h^{2}$, приходим к окончательной априорной оценке

$$
\|u\|_{0}^{2} \leqslant M_{1}\|\chi(x)\|_{0}^{2}+M_{2}\|f(x, y)\|_{0}^{2}
$$

где

$$
M_{1}=\frac{\gamma_{4} r^{2} h^{2}}{4 \varepsilon_{1}\left(2 r^{2}+2 h^{2}-\varepsilon_{2} r^{2} h^{2}\right)}, \quad M_{2}=\frac{r^{2} h^{2}}{4 \varepsilon_{2}\left(2 r^{2}+2 h^{2}-\varepsilon_{2} r^{2} h^{2}\right)} .
$$

Из априорной оценки (25) следует единственность регулярного решения задачи (3)-(6) при любой правой части $f(x, y) \in L_{2}(\Omega)$ и заданной функции $\psi(x) \in C^{3}[0, r / 2]$.

В заключение отметим, что априорные оценки решения задачи Трикоми для уравнения Лаврентьева-Бицадзе, подобные оценке (25), были получены в работах автора [3,4].

\section{СПИСОК ЛИТЕРАТУРЫ}

1. Алиханов A. А. Априорные оценки решений краевых задач для уравнений дробного порядка// Диффер. уравн. - 2010. - 46, № 5. - С. 658-664.

2. Бабенко К. И. К теории уравнений смешанного типа// Усп. мат. наук. - 1953. - 80, № 3. - С. 3-160.

3. Балкизов Ж. А., Сокуров A. А. Об априорной оценке решения задачи Трикоми для уравнения Лаврентьева-Бицадзе// Вестн. КРАУНЦ. Сер. физ.-мат. науки. - 2016. - 4-1 (16). - С. 15-20.

4. Балкизов Ж. А., Сокуров А. А. Об одном разностном методе решения задачи Трикоми для уравнения Лаврентьева-Бицадзе// Вестн. Самар. гос. техн. ун-та. Сер. физ.-мат. науки. - 2017. — 21, № 2. C. $221-235$.

5. Баранцев Р. Г. Лекции по трансзвуковой газовой динамике. - Ленинград: Изд-во Ленингр. гос. ун-та, 1965.

6. Берс Л. Математические вопросы дозвуковой и околозвуковой газовой динамики. - М.: ИЛ, 1961.

7. Бицадзе A. В. К проблеме уравнений смешанного типа. - М.: Изд-во АН СССР, 1953.

8. Бицадзе А. В. Некоторые классы уравнений в частных производных. - М.: Наука, 1981.

9. Бицадзе А. В. Уравнения смешанного типа. - М.: Изд-во АН СССР, 1959.

10. Девингталь Ю. В. О существовании и единственности решения одной задачи Ф. И. Франкля// Изв. вузов. Мат. - 1958. - № 2. - С. 39-51.

11. Жегалов В. И. Исследование краевых задач со смещением для уравнений смешанного типа/ дисс. на соиск. уч. степ. доктора физ.-мат. наук. - Новосибирск: Ин-т мат. им. С. Л. Соболева СО АН СССР, 1988.

12. Жегалов В. И. Краевая задача для уравнения смешанного типа с граничным условием на обеих характеристиках с разрывами на переходной линии// Уч. зап. Казан. гос. ун-та. - 1962. — 122, № 3. - C. $3-16$.

13. Жегалов В. И. К краевым задачам со смещениями для уравнения Лаврентьева-Бицадзе// Изв. вузов. Мат. - 1986. - № 3. - С. 61-64.

14. Кароль И. Л. К теории уравнений смешанного типа// Докл. АН СССР. - 1953. - 88, № 3. - С. 397400.

15. Кароль И. Л. Об одной краевой задаче для уравнения смешанного эллиптико-гиперболического типа// Докл. АН СССР. - 1953. - 88, № 2. - С. 197-200.

16. Крикунов Ю. М. Краевые задачи для модельных уравнений смешанного типа. - Казань: Изд-во Казан. ун-та, 1986.

17. Лаврентъев М. А., Бицадзе А. В. К проблеме уравнений смешанного типа// Докл. АН СССР. - 1950. - 70, № 3. - С. 373-376.

18. Нахушев А. М. Задачи со смещением для уравнений в частных производных. - М.: Наука, 2006. 
19. Нахушев А. М. К априорным оценкам для задач Трикоми и Дарбу// Диффер. уравн. - 1972. - 8, № 1. - C. 107-117.

20. Нахушев A. М. О некоторых краевых задачах для гиперболических уравнений и уравнений смешанного типа// Диффер. уравн. - 1969. - 5, № 1. - С. 44-59.

21. Нахушев A. М. Уравнения математической биологии. - М.: Наука, 1995.

22. Пулькин C. П. Задача Трикоми для общего уравнения Лаврентьева-Бицадзе// Докл. АН СССР. 1958. - 118, № 1. - С. 38-41.

23. Сабитов К. Б. К теории уравнений смешанного типа. - М.: Физматлит, 2014.

24. Смирнов M. М. Вырождающиеся эллиптические и гиперболические уравнения. - М.: Наука, 1966.

25. Смирнов М. М. Уравнения смешанного типа. - М.: Наука, 1970.

26. Солдатов А. П. Задачи типа Дирихле для уравнения Лаврентьева-Бицадзе// Диффер. уравн. 1994. - 30, № 11. - С. 2001-2009.

27. Солдатов А. П. Одномерные сингулярные операторы и краевые задачи теории функций. - М.: Высшая школа, 1991.

28. Трикоми Ф. Лекции по уравнениям в частных производных. - М.: ИЛ, 1957.

29. Трикоми $\Phi$. О линейных уравнениях второго порядка смешанного типа. - М.-Л.: Гостехиздат, 1947.

30. Франкль Ф. И. Избранные труды по газовой динамике. - М.: Наука, 1973.

31. Франкль Ф. И. О задачах Чаплыгина для смешанных до- и сверхзвуковых течений// Изв. АН СССР. Cep. мат. - 1945. - 9, № 2. - C. 121-142.

32. Gellerstedt $S$. Quelques problemes mixtes pour l'equation $y^{m} Z_{x x}+Z_{y y}=0 / /$ Ark. Math. Astr. Fysik. 1938. - 26A, № 3. - P. 1-32.

33. Gellerstedt $S$. Sur un problème aux limites pour une équation linéaire aux dérivées partielles du second ordre de tipe mixte. - Uppsala, 1935.

Балкизов Жираслан Анатольевич

Институт прикладной математики и автоматизации,

Кабардино-Балкарский научный центр Российской академии наук, Нальчик

E-mail: giraslan@yandex.ru 